Oracle Efficient Estimation and Forecasting with the Adaptive LASSO and the Adaptive Group LASSO in Vector Autoregressions

\author{
Anders Bredahl Kock and Laurent A.F. Callot
}

CREATES Research Paper 2012-38 


\title{
ORACLE EFFICIENT ESTIMATION AND FORECASTING WITH THE ADAPTIVE LASSO AND THE ADAPTIVE GROUP LASSO IN VECTOR AUTOREGRESSIONS
}

\author{
ANDERS BREDAHL KOCK AND LAURENT A.F. CALLOT \\ AARHUS UNIVERSITY AND CREATES
}

\begin{abstract}
We show that the adaptive Lasso (aLasso) and the adaptive group Lasso (agLasso) are oracle efficient in stationary vector autoregressions where the number of parameters per equation is smaller than the number of observations. In particular, this means that the parameters are estimated consistently at a $\sqrt{T}$ rate, that the truly zero parameters are classified as such asymptotically and that the non-zero parameters are estimated as efficiently as if only the relevant variables had been included in the model from the outset. The group adaptive Lasso differs from the adaptive Lasso by dividing the covariates into groups whose members are all relevant or all irrelevant. Both estimators have the property that they perform variable selection and estimation in one step.
\end{abstract}

We evaluate the forecasting accuracy of these estimators for a large set of macroeconomic variables. The Lasso is found to be the most precise procedure overall. The adaptive and the adaptive group Lasso are less stable but mostly perform at par with the common factor models.

Key words: Vector autoregression, VAR, adaptive Lasso, Group Lasso, Forecasting, Factor models, LSTAR.

JEL classifications: C32, C53, E17.

Date: September 1, 2012.

The first author would like to thank Timo Teräsvirta for guiding him through his Ph.D and for showing him the fascinating world of forecasting. However, the shortcomings of this paper are not Timo's responsibility. The authors also wish to thank the Centre for Research in the Econometric Analysis of Time Series (CREATES), funded by the Danish National Research Foundation, for financial support. 


\section{INTRODUCTION}

In recent years large data sets have become increasingly available and as a result techniques to handle these have been the object of considerable research. When building a model to explain the behavior of a variable it is not uncommon that the set of potential explanatory variables can be very large. Traditional techniques for model selection rely on a sequence of tests or the application of information criteria. However, neither of these is very useful when the the number of potential explanatory variables is large since the number of tests or information criteria to be calculated increases exponentially in the cardinality of the set of covariates. Hence, alternative routes have been investigated in and in particular regularized estimators have received a lot of attention in the statistics literature. The most prominent member of this class is the least absolute shrinkage and selection operator (Lasso) of Tibshirani (1996). Since its inception, the statistical properties of Lasso-type estimators have been studied intensively with particular focus on the oracle property. An estimator is said to possess the oracle property if i) it selects the correct sparsity pattern with probability tending to one (i.e leaves out all irrelevant variables and retains all relevant variables) and ii) estimates the non-zero coefficients with the same rate and asymptotic distribution as if only the relevant variables had been included in the model from the outset. Put differently, the oracle property guarantees that the estimator performs as well as if the true model had been revealed to the researcher in advance by an oracle.

A lot of research has been carried out investigating the oracle property of various shrinkage type estimators: bridge-type Knight and Fu (2000), SCAD Fan and Li (2001), adaptive Lasso Zou (2006), Bridge and Marginal Bridge Huang et al. (2008) and Sure independence screening Fan and Lv (2008). The working assumption in the literature is that even though the set of potential explanatory variables may be large (sometimes even considerably larger than the sample size) only a small subset of these variables are relevant 
for the task of explaining the left hand side variable, i.e. the model is sparse. Most focus has been on the cross sectional setting with either fixed or independently identically distributed covariates while much less attention has been paid to the case of dependent data. Some exceptions are Wang et al. (2007), Kock (2012) and Kock and Callot (2012). In this paper we further fill this gap by considering stationary vector autoregressive models of the type

$$
y_{t}=\sum_{i=1}^{p} B_{i} y_{t-i}+e_{t}
$$

where $y_{t}$ is $N \times 1$ and $e_{t}$ is i.i.d. with mean 0 and covariance matrix $\Sigma$. $B_{i}, 1 \leq i \leq p$ are the $N \times N$ parameter matrices. The properties of the model will be made precise in the next section.

It is likely that many entries in the $B_{i}$ matrices are equal to zero, i.e. they are sparse. This could be because of $p$ being larger than the true number of lags or that that there are gaps in the lag structure (e.g. $B_{1} \neq 0, B_{2}=B_{3}=$ 0 and $B_{4} \neq 0$ for quarterly data). Another reason could be that lags of a subset of the variables are irrelevant for the task of explaining another subset of variables which manifests itself by zero restrictions on certain entries of the $B_{i}, 1 \leq i \leq p$. Granger non-causality is an extreme case of this latter example. In the first part of this paper we show that the adaptive Lasso of Zou (2006) possesses the oracle property when applied to stationary vector autoregressions. Hence, it selects the correct sparsity pattern asymptotically and the non-zero parameters are estimated as precisely as if the true model had been known in advance and only the relevant variables had been included and estimated by least squares.

In equation (1) it is likely that zero parameters occur in groups. For example all lags of a specific length may be irrelevant resulting in $B_{i}=0$ for some $1 \leq i \leq N$. Alternatively, all lags of a certain variable may be irrelevant in explaining another variable. Utilizing this group structure may lead to improved (finite sample) performance of the Lasso. Hence, inspired 
by Wang and Leng (2008) we combine the group Lasso of Yuan and Lin (2006) with the adaptive Lasso to make use of this grouping structure. We show that the adaptive group Lasso possesses a variant of the oracle property if one correctly groups (a subset) of the potential explanatory variables.

Since vector autoregressions have been used extensively for forecasting an obvious question is how well the VAR performs in this respect when estimated by the Lasso, the adaptive Lasso or the adaptive group Lasso. In particular, we investigate the performances of these estimators for forecasting in large macroeconomic datasets. The benchmark models for this type of forecasting exercise are common factor models. The common factor approach is supported by a long tradition in macroeconomic theory of assuming that a small set of underlying variables drives the business cycle and are responsible for the bulk of the variation of macroeconomic time series. Stock and Watson (2002); Ludvigson and Ng (2009) inter alia document the strong forecasting power of these types of models for large US macroeconomic datasets. Motivated by this we shall compare the forecast accuracy of the Lasso type estimators to the one of factor models. A comparison to a simple linear autoregression of order one is also made. The potential gains in forecast accuracy from exploiting non-linearities in the data are investigated by also including the logistic smooth transition autoregression (LSTAR) of Teräsvirta (1994) into the comparison. Interestingly, it is found that the Lasso on average forecasts most precisely. The factor models show a very stable performance, while the forecast errors from the adaptive Lasso and the adaptive group Lasso are much more erratic.

In the next section we introduce the VAR model and some notation. Section 3 introduces the adaptive lasso and section 4 the adaptive group Lasso. Section 5 discusses the forecasting experiment and present the results. All proofs are relegated to the appendix. 


\section{Model AND NOTATION}

As mentioned in the introduction we are concerned with stationary VARs, meaning that all roots of $\left|I_{N}-\sum_{j=1}^{p} B_{j} z^{j}\right|$ lie outside the unit circle.

It is convenient to write the model in (1) as a standard regression model. To do so let $Z_{t}=\left(y_{t-1}^{\prime}, \ldots, y_{t-p}^{\prime}\right)^{\prime}$ be the $N p \times 1$ vector of explanatory variables at time $t$ in each equation $i=1, \ldots, N$ and $Z=\left(Z_{T}, \ldots, Z_{1}\right)^{\prime}$ the $T \times N p$ matrix of covariates. Set $X=I_{N} \otimes Z$ where $\otimes$ denotes the Kronecker product. Let $y_{i}=\left(y_{T, i}, \ldots, y_{1, i}\right)^{\prime}$ be the $T \times 1$ vector of observations on the $i$ th variable $(i=1, \ldots, N)$ and $\epsilon_{i}$ the corresponding vector of error terms for variable $i$. Defining $y=\left(y_{1}^{\prime}, \ldots, y_{N}^{\prime}\right)^{\prime}$ and $\epsilon=\left(\epsilon_{1}^{\prime}, \ldots, \epsilon_{N}^{\prime}\right)^{\prime}$ we may write (1) as

$$
y=X \beta^{*}+\epsilon
$$

where $\beta^{*}$ contains $N^{2} p$ parameters. It is this model we will estimate by adaptive and the adaptive group Lasso. We assume that $N$ and $p$ are fixed and independent of the sample size. In particular, we assume that the number of parameters per equation, $N p$, is less than the sample size $T$. For the setting where these quantities are allowed to diverge with the sample size we refer to Kock and Callot (2012) who however don’t consider the adaptive group Lasso.

While $\beta^{*}$ contains $N^{2} p$ parameters, only a subset of those might be relevant to model the dynamics of the vector $y$. The adaptive Lasso discussed in section 3 is able to discard the zero parameters and estimate the non-zero ones with an oracle efficient asymptotic distribution.

2.1. Further notation. Let $\mathcal{A}=\left\{i: \beta^{*} \neq 0\right\}$ index the set of nonzero $\beta_{i}^{*} \mathrm{~s}$ and let $|\mathcal{A}|$ be its cardinality. For any vector $x \in \mathbb{R}^{n}\|x\|=\sqrt{\sum_{i=1}^{n} x_{i}^{2}}$ denotes its euclidean norm. Furthermore, for any $A \subseteq\{1, \ldots, n\}, x_{A}$ denotes the vector consisting only of the elements indexed by $A$. Most often $n=N^{2} p$ 
in this paper. If $M$ is a quadratic matrix, $M_{A}$ denotes the submatrix of $M$ consisting of the rows and columns indexed by $A$. We let $\rightarrow_{d}$ and $\rightarrow_{p}$ denote convergence in distribution and probability, respectively.

Finally, $C=E\left(\frac{1}{T} Z^{\prime} Z\right)$ which is time independent by the stationarity assumption.

\section{The ADAptive Lasso}

As noted by Zhao and Yu (2007) the Lasso is only model selection consistent under rather restrictive assumptions which rule out highly dependent covariates as may be encountered in VAR models. Hence, we shall apply the adaptive Lasso, which was proposed by Zou (2006) as a solution to the lack of model selection consistency of the Lasso, to estimate the parameters in (2). The adaptive Lasso estimates $\beta^{*}$ by minimizing the following objective function.

$$
L_{T}(\beta)=\|y-X \beta\|^{2}+\lambda_{T} \sum_{i=1}^{N^{2} p} \hat{w}_{i}\left|\beta_{i}\right|
$$

where $\hat{w}_{i}$ is a set of weights such that $\hat{w}_{i}=\left|\hat{\beta}_{I, i}\right|^{-\gamma}, \gamma>0$ with $\hat{\beta}_{I}$ a $\sqrt{T}$ consistent (initial) estimator of $\beta^{*}$. We shall use the least squares estimator ${ }^{1}$. The most common choice of $\gamma$ is $\gamma=1$. $\lambda_{T}$ is a sequence whose properties determine the asymptotic properties of the adaptive Lasso. Note that the standard Lasso corresponds to the case of $\hat{w}_{i}=1$, i.e. all parameters receive an equal penalty. In other words the difference between the Lasso and its adaptive version is that the latter chooses its penalty terms more intelligently (adaptively): If $\beta_{i}^{*}=0$ for some $i=1, \ldots, N^{2} p$ the initial least squares estimator is likely to be close to zero and so $\hat{w}_{i}$ tends to be large resulting in a large penalty of $\beta_{i}$. Hence, the adaptive Lasso is more likely to correctly

\footnotetext{
${ }^{1}$ As already noted by Zou (2006) the initial estimator need not be $\sqrt{T}$-consistent. The assumptions made below can be altered such that theorems 1 and 2 still apply in the case where the initial estimator converges at a slower rate. However, we will not pursue this avenue any further here since we $d o$ have access to a $\sqrt{T}$-consistent consistent initial estimator.
} 
classify $\beta_{i}^{*}$ as zero. By a similar logic, the penalty on $\beta_{i}$ is relatively small when $\beta_{i}^{*} \neq 0$. As we shall see in the theorems to follow, these more intelligent weights result in an improved asymptotic performance of the adaptive Lasso compared to the regular Lasso.

The objective function (3) reveals the computational advantage of the (adaptive) lasso compared to e.g. information criteria since (3) is a convex optimization problem for which many efficient optimization procedures exist. Information criteria generally penalize model complexity by an $\ell_{0^{-}}$ penalty instead of the $\ell_{1}$-penalty used by lasso type estimators. It is exactly the switch from $\ell_{0}$ to $\ell_{1}$-penalty which yields the computational advantage enabling us to consider high dimensional problems which would be impossible or very hard to approach by means of $\ell_{0}$-penalization. As we will see next, the convex program (3) is not only fast to solve but its solution, the adaptive Lasso estimator, which we shall denote by $\hat{\beta}$, also possesses the oracle property.

\section{Assumptions}

1: $\epsilon_{i, t}$ has finite fourth moments for $i=1, \ldots, N$ and $t=1, \ldots, T$. Recall as well that $e_{t}=\left(\epsilon_{1, t}, \ldots, \epsilon_{N, t}\right)^{\prime}$ are mean zero iid vectors with covariance matrix $\Sigma$.

2: $C=E\left(\frac{1}{T} Z^{\prime} Z\right)$ is positive definite.

Assumption 1 is relatively standard and used to ensure that $\frac{1}{\sqrt{T}} X^{\prime} \epsilon$ converges in distribution to a gaussian random variable. But any assumption yielding this convergence will suffice for our purpose. Assumption 2 is reasonable since it simply rules out perfect collinearity because if $C$ would not be positive definite there would exist a nonzero $N p \times 1$ vector $v$ such that

$$
0=v^{\prime} C v=\frac{1}{T} E\left(v^{\prime} Z^{\prime} Z v\right)=\frac{1}{T} \sum_{t=1}^{T} E\left(v^{\prime} Z_{t}\right)^{2}
$$


implying that $v^{\prime} Z_{t}=0$ almost surely for $t=1, \ldots, T$ and hence that the covariates are linearly dependent. No procedure can be expected to distinguish between such variables, and assumption 2 rules out this situation.

We are now in a position to state our first theorem.

Theorem 1. Let assumptions 1 and 2 be satisfied and suppose that $\frac{\lambda_{T}}{\sqrt{T}} \rightarrow 0$ and $\frac{\lambda_{T}}{T^{1 / 2-\gamma / 2}} \rightarrow \infty$. Then $\hat{\beta}$ satisfies the following:

1. $\sqrt{T}$-consistency: $\left\|\sqrt{T}\left(\hat{\beta}-\beta^{*}\right)\right\|_{\ell_{2}} \in O_{p}(1)$

2. Oracle (i): $P\left(\hat{\beta}_{\mathcal{A}^{c}}=0\right) \rightarrow 1$

3. Oracle (ii): $\sqrt{T}\left(\hat{\beta}_{\mathcal{A}}-\beta_{\mathcal{A}}^{*}\right) \rightarrow_{d} N\left(0,\left[\left(I_{N} \otimes C\right)_{\mathcal{A}}\right]^{-1}[\Sigma \otimes C]_{\mathcal{A}}\left[\left(I_{N} \otimes C\right)_{\mathcal{A}}\right]^{-1}\right)$

The assumption $\frac{\lambda_{T}}{T^{1 / 2-\gamma / 2}} \rightarrow \infty$ is needed for the adaptive Lasso to shrink truly zero parameters to zero. It requires the penalty sequence $\lambda_{T}$ to increase sufficiently fast ${ }^{2}$. On the other hand, $\frac{\lambda_{T}}{\sqrt{T}} \rightarrow 0$ prevents $\lambda_{T}$ from increasing too fast. This is needed to prevent the adaptive Lasso from classifying nonzero parameters as zero.

Part 1 of Theorem 1 states that the adaptive Lasso converges at the usual $\sqrt{T}$-rate. This means that no $\hat{\beta}_{j}, j \in \mathcal{A}$ will be set equal to 0 since for all $j \in \mathcal{A}, \hat{\beta}_{j}$ converges in probability to $\beta_{j}^{*} \neq 0$. Part 2 is the first part of the oracle property: all truly zero parameters are set exactly equal to zero asymptotically. This is a strengthening of the consistency result in part 1 since this only ensures convergence in probability to 0 of $\hat{\beta}_{\mathcal{A}^{c}}$. Part 1 and 2 together imply that $P(\hat{\mathcal{A}}=\mathcal{A}) \rightarrow 1$. Part 3 states that the non-zero coefficients have the same asymptotic distribution as if the system in (2) had been estimated by least squares only including the relevant variables - i.e. only including the variables in the active set $\mathcal{A}$. In conclusion, the adaptive Lasso performs variable selection and estimation simultaneously and possesses the oracle property in the sense that it performs as well as if an oracle had revealed the true model prior to estimation.

\footnotetext{
${ }^{2}$ Strictly speaking $\lambda_{T}$ is only required to be increasing if $0<\gamma \leq 1$ but since $\gamma=1$ is the most common choice we shall use the word increasing without risk of confusion.
} 


\section{ADAPTIVE GROUP LASSO}

If certain groups of variables are either jointly zero or non-zero it may be useful to utilize this information to get more efficient (finite sample) estimates. For this reason Yuan and Lin (2006) introduced the group Lasso which penalizes different groups of variables differently. Later, Wang and Leng (2008) combined the ideas of the group Lasso and the adaptive Lasso into the adaptive group Lasso. We shall now show that the latter possesses a variant of the oracle property when used to estimate vector autoregressive models. Assume that the $N^{2} p \times 1$ parameter vector has been partitioned into $M$ disjoint groups, i.e. $\cup_{i=1}^{M} G_{i}=\left\{1, \ldots, N^{2} p\right\}$ and $G_{i} \cap G_{j}=\emptyset$ for $i \neq j$. A group $G_{i}$ is said to be active if at least one of the entries of $\beta_{G_{i}}^{*}$ is non-zero. Without any confusion with the previously introduced notation we shall denote the set of active groups by $\mathcal{A} \subseteq\{1, \ldots, M\} . \mathcal{G}=\cup_{i \in \mathcal{A}} G_{i} \subseteq$ $\left\{1, \ldots, N^{2} p\right\}$ denotes the union of the active groups.

The adaptive group LASSO estimates the parameters by minimizing the following objective function

$$
\tilde{L}_{T}(\beta)=\|y-X \beta\|^{2}+\lambda_{T} \sum_{j=1}^{M} \tilde{w}_{j}\left\|\beta_{G_{j}}\right\|
$$

where $\tilde{w}_{j}$ is a set of weights such that $\tilde{w}_{j}=\left\|\hat{\beta}_{I, G_{j}}\right\|^{-\gamma}, \gamma>0$ with $\hat{\beta}_{I, G_{j}}$ a $\sqrt{T}$-consistent estimator of $\beta^{*}$. As was the case the for the adaptive Lasso we will use the least squares estimator as initial estimator. Denote the group adaptive Lasso estimator by $\tilde{\beta}$. Note the difference with the objective function of the adaptive Lasso in (3): now the penalty is applied group-wise as opposed to being applied to each parameter individually. The economic motivation for this is that one might conjecture that either all variables in a specific group are relevant or none of them are. Imposing this (correct) restriction may increase efficiency. We shall investigate the empirical performance in terms of forecasting accuracy in the next section. But first we state the adaptive group Lasso equivalent of Theorem 1. 
Theorem 2. Let assumptions 1 and 2 be satisfied and suppose that $\frac{\lambda_{T}}{\sqrt{T}} \rightarrow 0$ and $\frac{\lambda_{T}}{T^{1 / 2-\gamma / 2}} \rightarrow \infty$. Then $\tilde{\beta}$ satisfies the following:

1. $\sqrt{T}$-consistency: $\left\|\sqrt{T}\left(\hat{\beta}-\beta^{*}\right)\right\|_{\ell_{2}} \in O_{p}(1)$

2. $\operatorname{Oracle}(i): P\left(\hat{\beta}_{\mathcal{G}^{c}}=0\right) \rightarrow 1$

3. Oracle (ii): $\sqrt{T}\left(\hat{\beta}_{\mathcal{G}}-\beta_{\mathcal{G}}^{*}\right) \rightarrow_{d} N\left(0,\left[\left(I_{N} \otimes C\right)_{\mathcal{G}}\right]^{-1}[\Sigma \otimes C]_{\mathcal{G}}\left[\left(I_{N} \otimes C\right)_{\mathcal{G}}\right]^{-1}\right)$

The assumptions underlying Theorem 2 are identical to the ones made to establish Theorem 1 and the intuition on the rate of increase of $\lambda_{T}$ is also the same: it must be large enough the shrink all inactive groups of parameters to zero while being small enough to avoid doing so for any active group of parameters.

Part 1 of Theorem 2 states the $\sqrt{T}$-consistency of the adaptive group Lasso. Hence, no relevant variables will be excluded asymptotically since $\tilde{\beta} \rightarrow_{p} \beta^{*} \neq 0$. Part 2 yields that all inactive groups are also classified to be inactive asymptotically. So all groups consisting only of parameters whose true value is zero will also be set exactly equal to zero with probability tending to one. However, note that this claim is not made about those parameters whose true value is zero but are (mistakenly) located in an active group. Their behavior is described in part 3 of the theorem: all parameters belonging to an active group are estimated with the same asymptotic distribution as if least squares had been applied to (2) only including variables belonging to $\mathcal{G}$. On the downside this means that the adaptive group Lasso only performs better than least squares including all variables if one is able to identify a group consisting only of zeros. On the other hand, the asymptotic distribution is equivalent to the one of least squares including all variables if one fails to do so and hence there is no efficiency loss. The empirical performance of the adaptive group Lasso estimator is investigated in the forecasting section. As we shall see there, many groups are found to be inactive in practice. 
4.1. Some limitations. As it stands, the oracle property sounds almost too good to be true - and in some sense it is. In a series of papers, Leeb and Pötscher (2005, 2008); Pötscher and Leeb (2009) shed critical light on consistent model selection procedures and shrinkage type estimators in particular. They point out that most results, including the ones in this paper, are for pointwise asymptotics (sometimes also referred to as fixed parameter asymptotics). The adaptive Lasso performs well in such a setting, but if uniform asymptotics are considered it may not be able to distinguish certain non-zero parameters from zero ones. In particular, the problematic regions are disks with radius proportional to $1 / \sqrt{T}$. Furthermore, even though the asymptotic distribution of the truly non-zero parameters is the same as if least squares had been applied only including the relevant variables one may find that the finite sample distributions can be highly bimodal - with mass at zero and in an interval around the true parameter value. Finally, using the mean square estimation error as loss function, the uniform (uniform over the parameter space) loss of any consistent model selection technique of the standard linear regression model may be shown to be infinite while the one of the least squares estimator can be shown to be finite.

\section{Forechisting}

In this section we investigate the empirical performance of the Lasso, the adaptive Lasso and the adaptive group Lasso in terms of forecasting macroeconomic variables with a large number of predictors. Vector autoregressive models have been used extensively for forecasting since their inception and are still a popular tool for this purpose in macroeconometrics. Hence, it is of interest to investigate whether novel estimation methods can lead to more precise forecasts in data rich settings.

5.1. The data. We use the data from Ludvigson and $\mathrm{Ng}$ (2009), which is itself an updated version of the data used in Stock and Watson (2002). The data set contains 131 U.S. monthly macroeconomic indicators, from 
January 1964 to December 2007. Detailed description of the series as well as the transformations required to make the series $\mathrm{I}(0)$ can be found in appendix A of Ludvigson and $\mathrm{Ng}$ (2009). The series fall in 8 broad economic categories:

(1) Output and Income (17 series)

(2) Labor market (32 series)

(3) Housing (10 series)

(4) Consumption, Orders and Inventory (14 series)

(5) Money and Credit (11 series)

(6) Bonds and Exchange rates (22 series)

(7) Prices (21 series)

(8) Stock market (4 series)

All variables are forecasted $h=1,3,6$, and 12 months ahead. The initial training sample uses data between 1964:33 and 1999:12 which amounts to 430 observations. We allow for a maximum of 2 lags per equation, which together with an intercept requires the estimation of 263 parameters per equation. All the parameters are estimated on the initial sample, then forecasts of $y_{t}$ at $\mathrm{t}=1999: 12+h, h=1,3,6,12$ are made. Parameters for all models are then re-estimated on data from 1964:3 to 2000:1 and forecasts computed at horizon $h$. This expanding window scheme is repeated until the final out of sample forecast is computed for $2007: 12$. At the one month horizon 96 forecasts are made and correspondingly less for the longer horizons.

The categories mentioned above serve as natural groups for the adaptive group Lasso and we shall indeed use these as candidate groups for this estimator. For each of the 131 series the relative mean square forecasts errors relative to the recursive forecasts ${ }^{4}$ of an unrestricted $\operatorname{VAR}(1)$ estimated by least squares are calculated ${ }^{5}$. Then the average of the relative mean square

\footnotetext{
${ }^{3}$ Two initial are lost during the transformation of the variables to $\mathrm{I}(0)$

${ }^{4}$ See the next subsection for a definition and discussion of recursive/iterated forecasts vs. direct forecasts.

${ }^{5}$ More precisely the lag length of the unrestricted VAR was chosen by BIC and it was always found to be one.
} 
forecast errors is calculated within each group resulting in one measure of forecast accuracy for each of the eight groups mentioned above.

5.2. Direct vs. recursive forecasts. The forecasts of the Lasso, adaptive Lasso and adaptive group Lasso are carried out directly as well as recursively. In the case of direct forecasts at horizon $h$, the estimated model is:

$$
y_{t+h}=\sum_{l=1}^{p} B_{l}^{h} y_{t-l+1}+\epsilon_{t+h}^{h}
$$

Where the superscript $h$ highlights the fact that a separate model is estimated for each horizon. The argument for direct forecasts is that they are tailored to the specific forecast horizon of interest. Furthermore, the absence of any sort of recursion makes direct forecasts relatively robust at the long forecast horizons.

Recursive forecasts are constructed iterating on a 1-step ahead VAR:

$$
y_{t}=\sum_{l=1}^{p} B_{l} y_{t-l}+\epsilon_{t}
$$

To construct the $h$ step ahead recursive forecasts, we first forecast $y_{t+1}$ using the model above and then use the forecasted value of $y_{t+1}$ to construct a forecast for $y_{t+2}$ and iterate until a forecast for $y_{t+h}$ is computed.

5.3. Implementation. Irrespective of the forecasts being direct or recursive the Lasso and the adaptive Lasso are estimated using the glmnet package for R 2.15, which implements the algorithm by Friedman et al. (2010). The value of $\lambda_{T}$ is selected by Bayesian Information Criterion (BIC). $\gamma$ is fixed at one and it is our experience that no gains can be achieved in terms of more precise forecasts by also searching over a grid of $\gamma \mathrm{s}$. The risk of overfitting in sample seems to be too high to justify such a search.

The adaptive group Lasso is estimated using the grplasso package, implementing the algorithm in Meier et al. (2008). Again $\lambda_{T}$ is selected by BIC while $\gamma$ is set to one. All the packages required for the computation 
of the results in this paper are publicly available at $\mathrm{CRAN}^{6}$, and the code is available upon request.

5.4. Competing models. The forecasts of the above mentioned procedures are compared to forecasts from common factor models, simple linear autoregressions, and smooth transition models.

For the common factor model we follow Stock and Watson (2002) in considering only direct forecasts. This avoids having to construct a forecasting model for the common factors in order to implement a recursive forecasting strategy. The forecasting equation for a given variable $y_{i}$ is given by:

$$
y_{t+h, i}=\alpha_{i}^{h}+\sum_{j=1}^{m} \hat{F}_{t-j+1}^{\prime} \beta_{i, j}^{h}+\sum_{j=1}^{p} \delta_{i, j}^{h} y_{t-j+1, i}+\epsilon_{t+h, i}^{h}
$$

The vector of common factors $\hat{F}_{t}$ and the parameters are estimated using a two step procedure. First the common factors $\hat{F}$ are estimated using principal component analysis on the training sample containing all 131 series. The number of principal components to retain for the second step is then selected and the parameters $\alpha_{i}^{h}, \beta_{i, j}^{h}$, and $\delta_{i, j}^{h}$ are estimated by least squares on the training sample.

We report results for models including 1 to 5 common factors and no lags of the common factors $(m=1)$ as well as a single lag of $y$. We experimented using lags of the common factors, but this didn't bring substantial nor consistent improvement to the forecasting accuracy of the model. These models are denoted $C F 1$ to $C F 5$ in the tables below. Furthermore, results for a common factor model where the number of factors is chosen by BIC are reported. The number of common factors searched over is one to five. The corresponding results are denoted $C F B I C$ in the tables.

The two univariate models considered are an $A R(1)$ and a Logistic Smooth Transition AutoRegressive (LSTAR) model. We consider direct forecasts for

\footnotetext{
$6_{\text {www.cran.r-project.org }}$
} 
both models. The forecasts from the $A R(1)$ model for $y_{t, i}$ are generated by

$$
y_{t+h, i}=\alpha_{i}^{h}+\beta_{i}^{h} y_{t, i}+\epsilon_{t+h, i}^{h}
$$

where the parameters are estimated by least squares. The forecasts of the LSTAR (see Teräsvirta (1994)) are created by the following model for variable $y_{t, i}$

$$
\begin{aligned}
y_{t+h, i} & =\left(\alpha_{1, i}^{h}+\beta_{1, i}^{h} y_{t, i}\right)\left(1-G\left(y_{t, i}, \gamma_{i}, \tau_{i}\right)\right) \\
& +\left(\alpha_{2, i}^{h}+\beta_{2, i}^{h} y_{t, i}\right)\left(G\left(y_{t, i}, \gamma_{i}, \tau_{i}\right)\right)+\epsilon_{t+h, i}^{h}
\end{aligned}
$$

where $G$ is the logistic function. For the LSTAR we use $y_{t}$ as the threshold variable. $\tau_{i}$ indicates the location oh the transition and $\gamma_{i}$ measures the speed of transition.

5.5. Insane forecasts. It is well known that in particular non-linear models may sometimes provide forecasts that are clearly unreasonable. Swanson and White (1995) suggests to weed out unreasonable forecasts by means of an insanity filter. We shall follow this suggestion by replacing a forecast by the most recent observation of the estimation window if it does not lie in the interval given by the most recent observation in the estimation window plus/minus three times the standard deviation of the data in the estimation window. As noted in Kock and Teräsvirta (2012) the particular choice of insanity filter is not overly important - what matters is that the insane forecasts are weeded out. To treat all forecast procedures on an equal footing the insanity filter is implemented for all procedures.

5.6. Results. Table 5.1 contains the relative mean square forecast errors (MSE) for each group of variables when averaged over all horizons $h=1,3,6$ and 12. The last column contains the average over all variable types. From this column it is seen that the Lasso gives the most precise forecasts on average. Whether one uses it to forecast recursively or directly is of no 
importance. The Lasso actually has a relative mean square forecast error below one for all groups of variables indicating its stability. Note also that except for the output and income group and the stock market group the most precise procedure is always the Lasso in either its recursive or direct variant. The plain Lasso actually outperforms its adaptive versions by a big margin. However, the relatively imprecise forecasts of these is to a high extent due to their poor performance when applied to the housing group. For this group the initial least squares estimator often gives wild initial parameter estimates resulting in unintelligent weights in the adaptive Lasso. However, this problem can be alleviated by using a regularized estimator such as the Lasso as initial estimator instead.

\section{[Insert table 5.1 here]}

In line with their strategy of finding common factors in the data set the factor models give reasonably precise forecasts for all types of variables resulting in mean square forecast error ratios below one for all groups. On the other hand, no gains seem to be made from applying BIC to select the number of factors as opposed to simply fixing the number of these.

As can be expected from a non-linear procedure like the LSTAR it performs very well for some series and quite poorly for others. This is in line with the commonly made observation that non-linear procedures are somewhat "risky" - a fact which can make them very useful in forecast combination schemes. To highlight this riskiness note that the LSTAR outperforms the plain $\operatorname{AR}(1)$ for five out of eight series while it still has a considerably larger relative mean square forecast error than common factor models and Lasso-type estimators due to its occasionally very imprecise forecasts.

Next, we shall further investigate the above overall findings by considering each forecast horizon and the composition of the models chosen by the Lassotype estimators in more detail.

[Insert table 5.2 here] 
The one month ahead forecast are reported in Table 5.2. Notice that since for 1-month ahead forecasts, recursive and direct forecasts are identical we do not make the distinction. Table 5.2 shows that common factor models as well as the Lasso and the adaptive Lasso deliver forecasts that are up to $50 \%$ more accurate than those obtained by a VAR estimated by least squares. The Lasso, and to a lesser extent the adaptive Lasso outperform common factor models for most groups. The adaptive group Lasso does perform quite poorly, faring worse than the benchmark VAR in 5 of the groups while being the best model for the Stock Market series. The two univariate forecasting models have very similar forecasting performances in most instances. The LSTAR model is less stable than the AR(1), being the best model for Bonds and Exchange Rates and the worst for Money and Credit.

[Insert table 5.3 here]

[Insert table 5.4 here]

To shed further light on these findings Table 5.3 reports the share of variables from a given group (in columns) retained in the equations for variables from another given group (in rows). The two leftmost entries of the first row in Table 5.3 should be read as: in the equations where the left-hand side variable belongs to the Output and Income group, $2.5 \%$ of the candidate explanatory variables from the Output and Income group were retained and $4 \%$ of the candidate explanatory variables belonging to the Labor Market group were retained. The boldfaced number is the largest share for a given row. Some striking differences between the behavior of the adaptive Lasso and the other two regularization estimators appear. The adaptive Lasso selects a large shares of variables belonging to the Consumption, Orders, Inventories series for most equations. The largest share selected by the other two estimators is often on the diagonal. Variables belonging to the same group as the left hand side variable are most often used as predictors. Another feature is that most of these shares are quite small, indicating the selected models are very sparse. This is confirmed by Table 5.4 which 
reports the average number of variables selected per group and for the whole equation. The models are often very sparse, the Lasso selecting between 6 and 10 out of the 262 candidate variables in each equations. The adaptive group Lasso often selects no variables at all in the housing equations, with an average of 0.188 variables per equation. Interestingly, this is also the group where this estimator performs worst.

[Insert table 5.5 here]

[Insert table 5.6 here]

Table 5.5 reports the results for the 3 -months ahead forecasts. The recursive and direct forecasts do not differ substantially, except for the VAR including all variables where the recursive model consistently outperforms the direct one. The Lasso consistently forecasts more precisely than every other procedure except for the Money and Credit group where it is not far behind $C F B I C$. The relative MSE are of the same order as those obtained at the 1-month horizon. The two adaptive estimators still perform very poorly for Housing and Bonds and Exchange rates. Similar observations can be made for 6-month ahead forecasts reported in Table 5.6, with one noticeable differences: the the common factor model is more often than previously the best estimator. In both tables 5.5 and 5.6, the LSTAR performs quite well for most groups and in general better than the $\operatorname{AR}(1)$. However, it fails badly for the Prices group at the 3 -month horizon.

[Insert table 5.7 here]

At the one year horizon (Table 5.7) the relative mean square errors of most procedures are even lower than at shorter horizons. The adaptive group Lasso delivers the most accurate forecasts for the Labor Market and Stock Market series while being close to the best procedure for most other groups. The Lasso outperforms the common factor models in three groups albeit not by a large margin. Common factors outperform every other procedure for three groups as well but the Lasso is a close second.

[Insert table 5.8 here] 
Table 5.8 is similar to table 5.3 for 12 month ahead forecasts. Since the Lasso uses the same model for each horizon only results for the direct forecasts are reported. The Lasso displays a pattern of selection different to that at the one month horizon (see table 5.3) selecting fewer variables on the diagonal and often selecting series belonging to the Housing group. The adaptive Lasso predominantly selects variables from the Money and Credit group, while it mostly selected Consumption, Orders, and Inventories at the one month horizon. The same finding is true for the adaptive group Lasso its selection pattern is now much more off-diagonal than previously. Finally, for the housing equation it never selects any variables resulting in forecasts that simply equal the mean of the estimation period. 


\section{Conclusion}

In this paper we have studied the properties of the adaptive Lasso and the adaptive group Lasso when applied to stationary vector autoregressions of fixed dimension. The adaptive Lasso was shown to possess the oracle property in the sense that all truly zero parameters will be classified as such asymptotically, while the estimators of the non-zero parameters have the same asymptotic distribution as if least squares had been used to the model only including the relevant variables.

Since many variables are naturally classified into groups of similar variables (like in the large macroeconomic dataset used in this paper) one may naturally ask the question whether certain groups of variables are relevant for the task of explaining another variable. For this reason the asymptotic properties of the adaptive group Lasso were investigated and it was shown that it possesses a version of the oracle property.

The performance of these two estimators in terms of forecast precision was investigated by comparing different forecasting procedures using the data by Ludvigson and $\mathrm{Ng}$ (2009). The plain Lasso was found to give the most precise forecasts on average while its adaptive variants had problems forecasting the housing series due to imprecise initial least squares estimates. The forecasts from the common factor models were relatively precise for all series while the non-linear LSTAR was much more unpredictable.

\section{Proof (APPEndix)}

Proof of Theorem 1: The proof is inspired by the proof of Theorem 2 in Zou (2006) and the proof of Theorem 2 in Kock (2012). Letting $\beta=\beta^{*}+\frac{u}{\sqrt{T}}$ the objective function (3) may also be written as

$$
L_{T}(u)=\left\|y-X\left(\beta^{*}+\frac{u}{\sqrt{T}}\right)\right\|^{2}+\lambda_{T} \sum_{i=1}^{N^{2} p} \hat{w}_{i}\left|\beta_{i}^{*}+\frac{u_{i}}{\sqrt{T}}\right|
$$

Let $\hat{u}=\arg \min L_{T}(u)$. It follows that $\hat{\beta}=\beta^{*}+\frac{\hat{u}}{\sqrt{T}}$ and so $\hat{u}=\sqrt{T}\left(\hat{\beta}-\beta^{*}\right)$. 
Next, define

$V_{T}(u)=L_{T}(u)-L_{T}(0)$

$$
=\left\|y-X\left(\beta^{*}+\frac{u}{\sqrt{T}}\right)\right\|^{2}-\left\|y-X \beta^{*}\right\|^{2}+\lambda_{T} \sum_{i=1}^{N^{2} p} \hat{w}_{i}\left(\left|\beta_{i}^{*}+\frac{u_{i}}{\sqrt{T}}\right|-\left|\beta_{i}^{*}\right|\right)
$$

(6)

$$
=u^{\prime} \frac{X^{\prime} X}{T} u-2 \frac{u^{\prime} X^{\prime} \epsilon}{\sqrt{T}}+\lambda_{T} \sum_{i=1}^{N^{2} p} \hat{w}_{i}\left(\left|\beta_{i}^{*}+\frac{u_{i}}{\sqrt{T}}\right|-\left|\beta_{i}^{*}\right|\right)
$$

By Theorem 11.2.1 in Brockwell and Davis (2009) it follows that $\frac{u^{\prime} X^{\prime} X u}{T} \rightarrow$ $u^{\prime}\left(I_{N} \otimes C\right) u$ in probability for any $u \in \mathbb{R}^{N^{2} p}$. Furthermore, it follows from Proposition 7.9 in Hamilton (1994) (see also expression 11.A.3 page 341 in Hamilton (1994)) that $\frac{X^{\prime} \epsilon}{\sqrt{T}} \rightarrow_{d} W \sim \mathcal{N}(0, \Sigma \otimes C)$. Hence,

$$
u^{\prime} \frac{X^{\prime} X}{T} u-2 \frac{u^{\prime} X^{\prime} \epsilon}{\sqrt{T}} \rightarrow_{d} u^{\prime}\left(I_{N} \otimes C\right) u-2 u^{\prime} W
$$

In addition, if $\beta_{i}^{*} \neq 0$

$$
\begin{aligned}
\lambda_{T} \hat{w}_{i}\left(\left|\beta_{i}^{*}+\frac{u_{i}}{\sqrt{T}}\right|-\left|\beta_{i}^{*}\right|\right) & =\lambda_{T}\left|\frac{1}{\hat{\beta}_{I, i}}\right|^{\gamma} \frac{u_{i}}{\sqrt{T}}\left(\left|\beta_{i}^{*}+\frac{u_{i}}{\sqrt{T}}\right|-\left|\beta_{i}^{*}\right|\right) /\left(\frac{u_{j}}{\sqrt{T}}\right) \\
& =\frac{\lambda_{T}}{T^{1 / 2}}\left|\frac{1}{\hat{\beta}_{I, i}}\right|^{\gamma} u_{i}\left(\left|\beta_{i}^{*}+\frac{u_{i}}{\sqrt{T}}\right|-\left|\beta_{i}^{*}\right|\right) /\left(\frac{u_{i}}{\sqrt{T}}\right) \\
& \rightarrow 0 \text { in probability }
\end{aligned}
$$

for every $u_{i} \in \mathbb{R}$ since (i): $\lambda_{T} / T^{1 / 2} \rightarrow 0$, (ii): $\left|1 / \hat{\beta}_{I, i}\right|^{\gamma} \rightarrow\left|1 / \beta_{i}^{*}\right|^{\gamma}<\infty$ in probability and

(iii): $u_{i}\left(\left|\beta_{i}^{*}+\frac{u_{i}}{\sqrt{T}}\right|-\left|\beta_{i}^{*}\right|\right) /\left(\frac{u_{i}}{\sqrt{T}}\right) \rightarrow u_{i} \operatorname{sign}\left(\beta_{i}^{*}\right)$.

If, on the other hand, $\beta_{i}^{*}=0$ 


$$
\begin{aligned}
\lambda_{T} \hat{w}_{i}\left(\left|\beta_{i}^{*}+\frac{u_{i}}{\sqrt{T}}\right|-\left|\beta_{i}^{*}\right|\right)= & \frac{\lambda_{T}}{T^{1 / 2}}\left|\frac{1}{\hat{\beta}_{I, i}}\right|^{\gamma}\left|u_{i}\right|=\frac{\lambda_{T}}{T^{1 / 2-\gamma / 2}}\left|\frac{1}{\sqrt{T} \hat{\beta}_{I, i}}\right|^{\gamma}\left|u_{i}\right| \\
& \rightarrow\left\{\begin{array}{l}
\infty \text { in probability if } u_{i} \neq 0 \\
0 \text { in probability if } u_{i}=0
\end{array}\right.
\end{aligned}
$$

since (i): $\frac{\lambda_{T}}{T^{1 / 2-\gamma / 2}} \rightarrow \infty$ and (ii): $\sqrt{T} \hat{\beta}_{i}$ is tight.

Using the convergence results (7)-(9) in (6) yields

$$
V_{T}(u) \rightarrow_{d} V(u)=\left\{\begin{array}{l}
u^{\prime}\left(I_{N} \otimes C\right) u-2 u^{\prime} W \text { if } u_{i}=0 \text { for all } i \in \mathcal{A}^{c} \\
\infty \text { if } u_{i} \neq 0 \text { for some } i \in \mathcal{A}^{c}
\end{array}\right.
$$

Since $V_{T}(u)$ is convex and $V(u)$ has a unique minimum it follows from Knight (1999) (or alternatively Knight and $\mathrm{Fu}(2000)$ ) that $\arg \min V_{T}(u) \rightarrow_{d} \arg \min V(u)$.

Hence,

$$
\begin{array}{r}
\hat{u}_{\mathcal{A}^{c}} \rightarrow{ }_{d} \delta_{0}^{\mathcal{A}^{c} \mid} \\
\hat{u}_{\mathcal{A}} \rightarrow_{d} N\left(0,\left[\left(I_{N} \otimes C\right)_{\mathcal{A}}\right]^{-1}[\Sigma \otimes C]_{\mathcal{A}}\left[\left(I_{N} \otimes C\right)_{\mathcal{A}}\right]^{-1}\right)
\end{array}
$$

where $\delta_{0}$ is the Dirac measure at 0 and $\left|\mathcal{A}^{c}\right|$ is the cardinality of $\mathcal{A}^{c}$ (hence, $\delta_{0}^{\left|\mathcal{A}^{c}\right|}$ is the $\left|\mathcal{A}^{c}\right|$-dimensional Dirac measure at 0$)$. Notice that (10) implies that $\hat{u}_{\mathcal{A}^{c}} \rightarrow 0$ in probability. An equivalent formulation of (10)-(11) is

$$
\begin{array}{r}
\sqrt{T}\left(\hat{\beta}_{\mathcal{A}^{c}}-\beta_{\mathcal{A}^{c}}^{*}\right) \rightarrow_{d} \delta_{0}^{\mathcal{A}^{c} \mid} \\
\sqrt{T}\left(\hat{\beta}_{\mathcal{A}}-\beta_{\mathcal{A}}^{*}\right) \rightarrow_{d} N\left(0,\left[\left(I_{N} \otimes C\right)_{\mathcal{A}}\right]^{-1}[\Sigma \otimes C]_{\mathcal{A}}\left[\left(I_{N} \otimes C\right)_{\mathcal{A}}\right]^{-1}\right)
\end{array}
$$

(12)-(13) yield the consistency part of the theorem at the rate of $\sqrt{T}$ for $\hat{\beta}$. (13) also yields the oracle efficient asymptotic distribution for $\hat{\beta}_{\mathcal{A}}$, 
i.e. part (3) of the theorem. It remains to show part (2) of the theorem; $P\left(\hat{\beta}_{\mathcal{A}^{c}}=0\right) \rightarrow 1$.

Assume $\hat{\beta}_{j} \neq 0$ for $j \in \mathcal{A}^{c}$. Then, letting $x_{j}$ denote the $j$ th column of $X$, it follows from the first order conditions

$$
2 x_{j}^{\prime}(y-X \hat{\beta})+\lambda_{T} \hat{w}_{j} \operatorname{sign}\left(\hat{\beta}_{j}\right)=0
$$

or equivalently,

$$
\frac{2 x_{j}^{\prime}(y-X \hat{\beta})}{T^{1 / 2}}+\frac{\lambda_{T} \hat{w}_{j} \operatorname{sign}\left(\hat{\beta}_{j}\right)}{T^{1 / 2}}=0
$$

First, consider the second term in (14)

$$
\left|\frac{\lambda_{T} \hat{w}_{j} \operatorname{sign}\left(\hat{\beta}_{j}\right)}{T^{1 / 2}}\right|=\frac{\lambda_{T} \hat{w}_{j}}{T^{1 / 2}}=\frac{\lambda_{T}}{T^{1 / 2-\gamma / 2}\left|T^{1 / 2} \hat{\beta}_{I, j}\right|^{\gamma}} \rightarrow \infty
$$

since $\sqrt{T} \hat{\beta}_{I, j}$ is tight. Regarding the first term in (14),

$$
\begin{aligned}
\frac{2 x_{j}^{\prime}(y-X \hat{\beta})}{T^{1 / 2}} & =\frac{2 x_{j}^{\prime}\left(\epsilon-X\left[\hat{\beta}-\beta^{*}\right]\right)}{T^{1 / 2}} \\
& =\frac{2 x_{j}^{\prime} \epsilon}{T^{1 / 2}}-\frac{2 x_{j}^{\prime} X}{T} \sqrt{T}\left[\hat{\beta}-\beta^{*}\right]
\end{aligned}
$$

Assuming $\beta_{j}$ is the coefficient to the $k$ th variable in the $i$ th equation (so the $j$ th column of $X$ is the $k$ th variable in the $i$ th equation) it follows from the same arguments as those preceding (6) that $\frac{x_{j}^{\prime} \epsilon}{T^{1 / 2}} \rightarrow_{d} N\left(0, \Sigma_{i i} C_{k k}\right)$. $\frac{x_{j}^{\prime} X}{T} \rightarrow_{p}\left(I_{N} \otimes C\right)_{j}$ where $\left(I_{N} \otimes C\right)_{j}$ is the $j$ th row of $\left(I_{N} \otimes C\right)$. Hence, $\frac{x_{j}^{\prime} \epsilon}{T^{1 / 2}}$ and $\frac{x_{j}^{\prime} X}{T}$ are tight. The same is the case for $\sqrt{T}\left[\hat{\beta}-\beta^{*}\right]$ since it converges weakly by (12)-(13). Taken together, $\frac{2 x_{j}^{\prime}(y-X \hat{\beta})}{T^{1 / 2}}$ is tight and so

$$
P\left(\hat{\beta}_{j} \neq 0\right) \leq P\left(\frac{2 x_{j}^{\prime}(y-X \hat{\beta})}{T^{1 / 2}}+\frac{\lambda_{T} \hat{w}_{j} \operatorname{sign}\left(\hat{\beta}_{j}\right)}{T^{1 / 2}}=0\right) \rightarrow 0
$$


Proof of Theorem 2. The idea of the proof is similar to the one of Theorem

1. Letting $\beta=\beta^{*}+\frac{u}{\sqrt{T}}$ the objective function (4) may also be written as

$$
\tilde{L}_{T}(u)=\left\|y-X\left(\beta^{*}+\frac{u}{\sqrt{T}}\right)\right\|^{2}+\lambda_{T} \sum_{i=1}^{M} \tilde{w}_{i}\left\|\beta_{G_{i}}^{*}+\frac{u_{G_{i}}}{\sqrt{T}}\right\|
$$

Let $\tilde{u}=\arg \min L_{T}(u)$. It follows that $\tilde{\beta}=\beta^{*}+\frac{\tilde{u}}{\sqrt{T}}$ and so $\tilde{u}=\sqrt{T}\left(\tilde{\beta}-\beta^{*}\right)$.

Next, define

$$
\begin{aligned}
\tilde{V}_{T}(u) & =\tilde{L}_{T}(u)-\tilde{L}_{T}(0) \\
& =\left\|y-X\left(\beta^{*}+\frac{u}{\sqrt{T}}\right)\right\|^{2}-\left\|y-X \beta^{*}\right\|^{2}+\lambda_{T} \sum_{i=1}^{M} \tilde{w}_{i}\left(\left\|\beta_{G_{i}}^{*}+\frac{u_{G_{i}}}{\sqrt{T}}\right\|-\left\|\beta_{G_{i}}^{*}\right\|\right)
\end{aligned}
$$

$$
=u^{\prime} \frac{X^{\prime} X}{T} u-2 \frac{u^{\prime} X^{\prime} \epsilon}{\sqrt{T}}+\lambda_{T} \sum_{i=1}^{M} \tilde{w}_{i}\left(\left\|\beta_{G_{i}}^{*}+\frac{u_{G_{i}}}{\sqrt{T}}\right\|-\left\|\beta_{G_{i}}^{*}\right\|\right)
$$

By Theorem 11.2.1 in Brockwell and Davis (2009) it follows that $\frac{u^{\prime} X^{\prime} X u}{T} \rightarrow_{p}$ $u^{\prime}\left(I_{N} \otimes C\right) u$ in probability for any $u \in \mathbb{R}^{N^{2} p}$. Furthermore, it follows from Proposition 7.9 in Hamilton (1994) (see also expression 11.A.3 page 341 in Hamilton (1994)) that $\frac{X^{\prime} \epsilon}{\sqrt{T}} \rightarrow_{d} W$ where $W \sim \mathcal{N}(0, \Sigma \otimes C)$. Hence,

$$
u^{\prime} \frac{X^{\prime} X}{T} u-2 \frac{u^{\prime} X^{\prime} \epsilon}{\sqrt{T}} \rightarrow_{d} u^{\prime}\left(I_{N} \otimes C\right) u-2 u^{\prime} W
$$

In addition, if $\beta_{G_{i}}^{*} \neq 0$, it follows by continuity of the norm that

$$
\begin{aligned}
\left|\lambda_{T} \tilde{w}_{i}\left(\left\|\beta_{G_{i}}^{*}+\frac{u_{G_{i}}}{\sqrt{T}}\right\|-\left\|\beta_{G_{i}}^{*}\right\|\right)\right| & \leq \lambda_{T} \tilde{w}_{i}\left\|\frac{u_{G_{i}}}{\sqrt{T}}\right\|=\frac{\lambda_{T}}{\sqrt{T}} \frac{\left\|u_{G_{i}}\right\|}{\left\|\hat{\beta}_{I, G_{i}}\right\|^{\gamma}} \\
& \rightarrow 0 \text { in probability }
\end{aligned}
$$

since (i): $\lambda_{T} / T^{1 / 2} \rightarrow 0$ and (ii): $\frac{1}{\left\|\hat{\beta}_{I, G_{i}}\right\|^{\gamma}} \rightarrow \frac{1}{\left\|\beta_{G_{i}}^{*}\right\|^{\gamma}}<\infty$ in probability. If, on the other hand, $\beta_{G_{i}}^{*}=0$ 


$$
\begin{aligned}
& \lambda_{T} \tilde{w}_{i}\left(\left\|\beta_{G_{i}}^{*}+\frac{u_{G_{i}}}{\sqrt{T}}\right\|-\left\|\beta_{G_{i}}^{*}\right\|\right)=\lambda_{T} \tilde{w}_{i}\left\|\frac{u_{G_{i}}}{\sqrt{T}}\right\|=\frac{\lambda_{T}}{T^{1 / 2-\gamma / 2}} \frac{\left\|u_{G_{i}}\right\|}{\left\|\sqrt{T} \hat{\beta}_{I, G_{i}}\right\|^{\gamma}} \\
& \rightarrow\left\{\begin{array}{l}
\infty \text { in probability if } u_{G_{i}} \neq 0 \\
0 \text { in probability if } u_{G_{i}}=0
\end{array}\right.
\end{aligned}
$$

since (i): $\frac{\lambda_{T}}{T^{1 / 2-\gamma / 2}} \rightarrow \infty$ and (ii): $\sqrt{T} \hat{\beta}_{I, G_{i}}$ is tight.

Using the convergence results (17)-(19) in (16)

$$
\tilde{V}_{T}(u) \rightarrow_{d} \tilde{V}(u)=\left\{\begin{array}{l}
u^{\prime}\left(I_{N} \otimes C\right) u-2 u^{\prime} W \text { if } u_{G_{i}}=0 \text { for all } i \in \mathcal{A}^{c} \\
\infty \text { if } u_{G_{i}} \neq 0 \text { for some } i \in \mathcal{A}^{c}
\end{array}\right.
$$

Since $\tilde{V}_{T}(u)$ is convex and $\tilde{V}(u)$ has a unique minimum it follows from Knight (1999) (or alternatively Knight and $\mathrm{Fu}(2000)$ ) that $\arg \min \tilde{V}_{T}(u) \rightarrow_{d} \arg \min \tilde{V}(u)$.

Hence,

$$
\begin{array}{r}
\tilde{u}_{\mathcal{G}^{c}} \rightarrow_{d} \delta_{0}^{\left|\mathcal{G}^{c}\right|} \\
\tilde{u}_{\mathcal{G}} \rightarrow_{d} N\left(0,\left[\left(I_{N} \otimes C\right)_{\mathcal{G}}\right]^{-1}[\Sigma \otimes C]_{\mathcal{G}}\left[\left(I_{N} \otimes C\right)_{\mathcal{G}}\right]^{-1}\right)
\end{array}
$$

where $\delta_{0}$ is the Dirac measure at 0 and $\left|\mathcal{G}^{c}\right|$ is the cardinality of $\mathcal{G}^{c}$. Notice that (20) implies that $\tilde{u}_{\mathcal{G}^{c}} \rightarrow 0$ in probability. An equivalent formulation of (20)-(21) is

$$
\begin{array}{r}
\sqrt{T}\left(\tilde{\beta}_{\mathcal{G}^{c}}-\beta_{\mathcal{G}^{c}}^{*}\right) \rightarrow_{d} \delta_{0}^{\left|\mathcal{G}^{c}\right|} \\
\sqrt{T}\left(\tilde{\beta}_{\mathcal{G}}-\beta_{\mathcal{G}}^{*}\right) \rightarrow_{d} N\left(0,\left[\left(I_{N} \otimes C\right)_{\mathcal{G}}\right]^{-1}[\Sigma \otimes C]_{\mathcal{G}}\left[\left(I_{N} \otimes C\right)_{\mathcal{G}}\right]^{-1}\right)
\end{array}
$$

(22)-(23) yield the consistency part of the theorem at the rate of $\sqrt{T}$ for $\tilde{\beta}$. (23) also yields the asymptotic distribution for $\tilde{\beta}_{\mathcal{G}}$, i.e. part 3 of the theorem. It remains to show part 2 of the theorem; $P\left(\tilde{\beta}_{\mathcal{G}^{c}}=0\right) \rightarrow 1$. 
Assume $\tilde{\beta}_{G_{i}} \neq 0$ for $i \in \mathcal{A}^{c}$. Then all entries $\tilde{\beta}_{G_{i}, j}, 1 \leq j \leq\left|G_{i}\right|$ satisfy the first order condition

$$
2 x_{j}^{\prime}(y-X \tilde{\beta})+\lambda_{T} \tilde{w}_{i}\left\|\tilde{\beta}_{G_{i}}\right\|^{-1} \tilde{\beta}_{G_{i}, j}=0
$$

or equivalently,

$$
\frac{2 x_{j}^{\prime}(y-X \tilde{\beta})}{T^{1 / 2}}+\frac{\lambda_{T} \tilde{w}_{i}\left\|\tilde{\beta}_{G_{i}}\right\|^{-1} \tilde{\beta}_{G_{i}, j}}{T^{1 / 2}}=0
$$

This also implies

$$
\max _{1 \leq j \leq\left|G_{i}\right|}\left|\frac{2 x_{j}^{\prime}(y-X \tilde{\beta})}{T^{1 / 2}}\right|=\max _{1 \leq j \leq\left|G_{i}\right|}\left|\frac{\lambda_{T} \tilde{w}_{i}\left\|\tilde{\beta}_{G_{i}}\right\|^{-1} \tilde{\beta}_{G_{i}, j}}{T^{1 / 2}}\right|
$$

First, consider the right hand side of (24). To this end note that

$\frac{\max _{1 \leq j \leq\left|G_{i}\right|}\left|\tilde{\beta}_{G_{i}, j}\right|}{\| \tilde{\beta}_{G_{i}} \mid} \geq \frac{\max _{1 \leq j \leq\left|G_{i}\right|}\left|\tilde{\beta}_{G_{i}, j}\right|}{\sum_{j=1}^{\left|G_{i}\right|}\left|\tilde{\beta}_{G_{i}, j}\right|} \geq \frac{\max _{1 \leq j \leq\left|G_{i}\right|}\left|\tilde{\beta}_{G_{i}, j}\right|}{\left|G_{i}\right| \max _{1 \leq j \leq\left|G_{i}\right|}\left|\tilde{\beta}_{G_{i}, j}\right|}=\frac{1}{\left|G_{i}\right|}$

This implies

$$
\begin{aligned}
\max _{1 \leq j \leq\left|G_{i}\right|}\left|\frac{\lambda_{T} \tilde{w}_{i}\left\|\tilde{\beta}_{G_{i}}\right\|^{-1} \tilde{\beta}_{G_{i}, j}}{T^{1 / 2}}\right| & =\frac{\lambda_{T} \tilde{w}_{i}}{T^{1 / 2}} \frac{\max _{1 \leq j \leq\left|G_{i}\right|}\left|\tilde{\beta}_{G_{i}, j}\right|}{\left\|\tilde{\beta}_{G_{j}}\right\|} \\
& \geq \frac{\lambda_{T}}{T^{1 / 2-\gamma / 2}} \frac{1}{\left\|T^{1 / 2} \hat{\beta}_{I, G_{i}}\right\|^{\gamma}} \frac{1}{\left|G_{i}\right|} \rightarrow_{p} \infty
\end{aligned}
$$

since $\sqrt{T} \hat{\beta}_{I, G_{i}}$ is tight.

Regarding the left hand side in (24),

$$
\begin{aligned}
\frac{2 x_{j}^{\prime}(y-X \tilde{\beta})}{T^{1 / 2}} & =\frac{2 x_{j}^{\prime}\left(\epsilon-X\left[\tilde{\beta}-\beta^{*}\right]\right)}{T^{1 / 2}} \\
& =\frac{2 x_{j}^{\prime} \epsilon}{T^{1 / 2}}-\frac{2 x_{j}^{\prime} X}{T} \sqrt{T}\left[\tilde{\beta}-\beta^{*}\right]
\end{aligned}
$$


Assuming $\beta_{j}$ is a coefficient to the $k$ th variable in the $i$ th equation it follows from the same arguments as those preceding (16) that $\frac{x_{j}^{\prime} \epsilon}{T^{1 / 2}} \rightarrow_{d}$ $N\left(0, \Sigma_{i i}^{2} C_{k k}\right) . \frac{x_{j}^{\prime} X}{T} \rightarrow_{p}\left(I_{N} \otimes C\right)_{j}$ where $\left(I_{N} \otimes C\right)_{j}$ is the $i$ th row of $\left(I_{N} \otimes C\right)$. Hence, $\frac{x_{j}^{\prime} \epsilon}{T^{1 / 2}}$ and $\frac{x_{j}^{\prime} X}{T}$ are tight. The same is the case for $\sqrt{T}\left[\tilde{\beta}-\beta^{*}\right]$ since it converges weakly by (22)-(23). Taken together, $\frac{2 x_{j}^{\prime}(y-X \tilde{\beta})}{T^{1 / 2}}$ is tight for all $j=1, \ldots, N^{2} p$. Furthermore,

$$
\begin{aligned}
P\left(\max _{1 \leq j \leq\left|G_{i}\right|}\left|\frac{2 x_{j}^{\prime}(y-X \tilde{\beta})}{T^{1 / 2}}\right|>K\right) & \leq\left|G_{i}\right| \max _{1 \leq j \leq\left|G_{i}\right|} P\left(\left|\frac{2 x_{j}^{\prime}(y-X \tilde{\beta})}{T^{1 / 2}}\right|>K\right) \\
& \leq\left|G_{i}\right| \max _{1 \leq j \leq\left|G_{i}\right| T \geq 1} \sup _{T \geq 1}\left(\left|\frac{2 x_{j}^{\prime}(y-X \tilde{\beta})}{T^{1 / 2}}\right|>K\right)
\end{aligned}
$$

implies

$\sup _{T \geq 1} P\left(\max _{1 \leq j \leq\left|G_{i}\right|}\left|\frac{2 x_{j}^{\prime}(y-X \tilde{\beta})}{T^{1 / 2}}\right|>K\right) \leq\left|G_{i}\right| \max _{1 \leq j \leq\left|G_{i}\right| T \geq 1} \sup _{T \geq 1}\left(\left|\frac{2 x_{j}^{\prime}(y-X \tilde{\beta})}{T^{1 / 2}}\right|>K\right)$

And so, for any $\delta>0$ by choosing $K$ sufficiently large it follows from the tightness of $\frac{2 x_{j}^{\prime}(y-X \tilde{\beta})}{T^{1 / 2}}, j \in G_{i}$ that

$$
\inf _{T \geq 1} P\left(\max _{1 \leq j \leq\left|G_{i}\right|}\left|\frac{2 x_{j}^{\prime}(y-X \tilde{\beta})}{T^{1 / 2}}\right| \leq K\right) \geq 1-\delta
$$

Since the right hand side in (24) will be larger than $K$ from a certain step and onwards it follows that $P\left(\tilde{\beta}_{G_{i}}=0\right) \rightarrow 1$. 


\section{REFERENCES}

Brockwell, P. And R. Davis (2009): Time series: theory and methods, Springer Verlag.

FAN, J. AND R. Li (2001): "Variable selection via nonconcave penalized likelihood and its oracle properties," Journal of the American Statistical Association, 96, 1348-1360.

FAN, J. AND J. LV (2008): "Sure independence screening for ultrahigh dimensional feature space," Journal of the Royal Statistical Society: Series B (Statistical Methodology), 70, 849-911.

Friedman, J., T. Hastie, and R. Tibshirani (2010): "Regularization paths for generalized linear models via coordinate descent," Journal of statistical software, 33,1 .

Hamilton, J. (1994): Time series analysis, vol. 2, Cambridge Univ Press.

Huang, J., J. Horowitz, and S. Ma (2008): “Asymptotic properties of bridge estimators in sparse high-dimensional regression models," The Annals of Statistics, 36, 587-613.

Knight, K. (1999): "Epi-convergence in distribution and stochastic equisemicontinuity," Unpublished manuscript.

Knight, K. AND W. Fu (2000): "Asymptotics for lasso-type estimators," Annals of Statistics, 1356-1378.

Kock, A. And L. Callot (2012): "Oracle Inequalities for High Dimensional Vector Autoregressions," CREATES working paper 2012-05.

Kock, A. And T. Teräsvirta (2012): "Forecasting Macroeconomic Variables using Neural Network Models and Three Automated Model Selection Techniques," CREATES Research Papers.

Kock, A. B. (2012): "Consistent and conservative model selection in stationary and non-stationary autoregressions," Submitted.

Leeb, H. AND B. Pötscher (2005): "Model selection and inference: Facts and fiction," Econometric Theory, 21, 21-59. 
(2008): "Sparse estimators and the oracle property, or the return of Hodges' estimator," Journal of Econometrics, 142, 201-211.

Ludvigson, S. AND S. NG (2009): "Macro factors in bond risk premia," Review of Financial Studies, 22, 5027-5067.

Meier, L., S. Van De Geer, and P. Bühlmann (2008): "The group lasso for logistic regression," Journal of the Royal Statistical Society: Series B (Statistical Methodology), 70, 53-71.

PÖtscher, B. AND H. Leeb (2009): "On the distribution of penalized maximum likelihood estimators: The LASSO, SCAD, and thresholding," Journal of Multivariate Analysis, 100, 2065-2082.

Stock, J. And M. Watson (2002): "Forecasting using principal components from a large number of predictors," Journal of the American Statistical Association, 97, 1167-1179.

Swanson, N. And H. White (1995): "A model-selection approach to assessing the information in the term structure using linear models and artificial neural networks," Journal of Business 8 Economic Statistics, 265-275.

TerÄsvirta, T. (1994): "Specification, estimation, and evaluation of smooth transition autoregressive models," Journal of the American Statistical Association, 208-218.

Tibshirani, R. (1996): "Regression shrinkage and selection via the lasso," Journal of the Royal Statistical Society. Series B (Methodological), 267288.

Wang, H. And C. Leng (2008): "A note on adaptive group lasso," Computational Statistics \& Data Analysis, 52, 5277-5286.

Wang, H., G. Li, And C. L. Tsai (2007): "Regression coefficient and autoregressive order shrinkage and selection via the lasso," Journal of the Royal Statistical Society: Series B (Statistical Methodology), 69, 63-78.

YUAN, M. AND Y. Lin (2006): "Model selection and estimation in regression with grouped variables," Journal of the Royal Statistical Society: 
Series B (Statistical Methodology), 68, 49-67.

Zhao, P. And B. Yu (2007): "On model selection consistency of Lasso," Journal of Machine Learning Research, 7, 2541.

Zou, H. (2006): "The adaptive lasso and its oracle properties," Journal of the American Statistical Association, 101, 1418-1429. 


\begin{tabular}{|c|c|c|c|c|c|c|c|c|c|}
\hline & $\begin{array}{l}\text { Output } \\
\text { and } \\
\text { Income }\end{array}$ & $\begin{array}{l}\text { Labor } \\
\text { Market }\end{array}$ & Housing & $\begin{array}{l}\text { Consumption } \\
\text { Orders } \\
\text { Inventories }\end{array}$ & $\begin{array}{l}\text { Money } \\
\text { and } \\
\text { Credit }\end{array}$ & $\begin{array}{c}\text { Bonds and } \\
\text { Exchange } \\
\text { Rates }\end{array}$ & Prices & $\begin{array}{c}\text { Stock } \\
\text { Market }\end{array}$ & Total \\
\hline \multicolumn{10}{|c|}{ Recursive Forecasts } \\
\hline Lasso & 0.537 & 0.593 & 0.575 & 0.540 & 0.503 & 0.450 & 0.549 & 0.754 & 0.563 \\
\hline aLasso & 0.579 & 0.696 & 5.000 & 0.601 & 0.503 & 1.143 & 0.583 & 0.762 & 1.234 \\
\hline agLasso & 0.615 & 0.791 & 6.174 & 0.831 & 0.520 & 1.468 & 0.650 & 0.749 & 1.475 \\
\hline \multicolumn{10}{|c|}{ Direct Forecasts } \\
\hline OLS-VAR & 1.281 & 1.346 & 1.063 & 1.154 & 0.922 & 1.382 & 0.948 & 1.862 & 1.245 \\
\hline Lasso & 0.556 & 0.582 & 0.511 & 0.575 & 0.499 & 0.480 & 0.553 & 0.750 & 0.563 \\
\hline aLasso & 0.616 & 0.740 & 5.138 & 0.676 & 0.512 & 1.096 & 0.602 & 0.771 & 1.269 \\
\hline agLasso & 0.614 & 0.790 & 6.152 & 0.830 & 0.521 & 1.469 & 0.650 & 0.749 & 1.472 \\
\hline \multicolumn{10}{|c|}{ Factor model forecasts } \\
\hline CF 1 & 0.545 & 0.610 & 0.819 & 0.654 & 0.520 & 0.516 & 0.610 & 0.803 & 0.635 \\
\hline $\mathrm{CF} 2$ & 0.539 & 0.605 & 0.859 & 0.635 & 0.520 & 0.497 & 0.595 & 0.845 & 0.637 \\
\hline $\mathrm{CF} 3$ & 0.531 & 0.621 & 0.859 & 0.603 & 0.521 & 0.494 & 0.589 & 0.847 & 0.633 \\
\hline $\mathrm{CF} 4$ & 0.528 & 0.618 & 0.827 & 0.591 & 0.522 & 0.494 & 0.587 & 0.839 & 0.626 \\
\hline $\mathrm{CF} 5$ & 0.536 & 0.619 & 0.824 & 0.603 & 0.522 & 0.496 & 0.592 & 0.844 & 0.629 \\
\hline $\mathrm{CF} \mathrm{BIC}$ & 0.541 & 0.610 & 0.853 & 0.651 & 0.544 & 0.569 & 0.609 & 0.830 & 0.651 \\
\hline \multicolumn{10}{|c|}{ Univariate forecasts } \\
\hline LSTAR & 0.632 & 0.592 & 0.812 & 0.613 & 1.854 & 0.477 & 4.590 & 0.886 & 1.307 \\
\hline $\operatorname{AR}(1)$ & 0.915 & 0.801 & 0.771 & 0.842 & 0.916 & 0.710 & 1.129 & 1.357 & 0.930 \\
\hline
\end{tabular}

TABLE 5.1. MSE relative to the recursive VAR MSE, average across all forecast horizons. Lowest relative MSE in bold.

\begin{tabular}{lcccccccc}
\hline & $\begin{array}{c}\text { Output } \\
\text { and } \\
\text { Income }\end{array}$ & $\begin{array}{c}\text { Labor } \\
\text { Market }\end{array}$ & Housing & $\begin{array}{c}\text { Consumption } \\
\text { Orders } \\
\text { Inventories }\end{array}$ & $\begin{array}{c}\text { Money } \\
\text { and } \\
\text { Credit }\end{array}$ & $\begin{array}{c}\text { Bonds and } \\
\text { Exchange } \\
\text { Rates }\end{array}$ & Prices & $\begin{array}{c}\text { Stock } \\
\text { Market }\end{array}$ \\
\hline Lasso & 0.6391 & $\mathbf{0 . 5 5 6 6}$ & $\mathbf{0 . 5 9 0 9}$ & $\mathbf{0 . 5 6 6 9}$ & $\mathbf{0 . 6 6 7 5}$ & 0.3620 & $\mathbf{0 . 7 0 7 1}$ & 0.5206 \\
aLasso & 0.7143 & 0.7787 & 8.2574 & 0.6486 & 0.7059 & 1.8806 & 0.8473 & 0.5499 \\
agLasso & 0.8537 & 1.0761 & 10.6095 & 1.3140 & 0.7324 & 2.8551 & 1.0303 & $\mathbf{0 . 5 1 7 2}$ \\
CF 1 & 0.6001 & 0.6833 & 0.8246 & 0.7527 & 0.8166 & 0.5432 & 0.8743 & 0.5851 \\
CF 2 & 0.5740 & 0.6734 & 0.8302 & 0.7182 & 0.8177 & 0.5263 & 0.8518 & 0.5813 \\
CF 3 & $\mathbf{0 . 5 5 5 0}$ & 0.7175 & 0.8252 & 0.6486 & 0.8173 & 0.5185 & 0.8243 & 0.5887 \\
CF 4 & 0.5709 & 0.7361 & 0.8294 & 0.6380 & 0.8165 & 0.5320 & 0.8144 & 0.5774 \\
CF 5 & 0.5691 & 0.7328 & 0.8315 & 0.6737 & 0.8183 & 0.5393 & 0.8263 & 0.5848 \\
CF BIC & 0.6007 & 0.6834 & 0.8244 & 0.7529 & 0.8164 & 0.5432 & 0.8744 & 0.5852 \\
& & \multicolumn{7}{c}{ Univariate forecasts } \\
LSTAR & 0.8742 & 0.6624 & 0.7779 & 0.7035 & 4.3938 & $\mathbf{0 . 3 5 9 7}$ & 1.2135 & 0.6285 \\
AR(1) & 0.9615 & 0.6772 & 0.7726 & 0.7351 & 1.3980 & 0.3701 & 1.5042 & 0.6431 \\
\hline
\end{tabular}

TABLE 5.2. MSE relative to the recursive VAR MSE. 1 step ahead forecasts, 96 forecasts 


\begin{tabular}{|c|c|c|c|c|c|c|c|c|}
\hline & $\begin{array}{l}\text { Output } \\
\text { and } \\
\text { Income }\end{array}$ & $\begin{array}{c}\text { Labor } \\
\text { Market }\end{array}$ & Housing & $\begin{array}{l}\text { Consumption } \\
\text { Orders } \\
\text { Inventories }\end{array}$ & $\begin{array}{l}\text { Money } \\
\text { and } \\
\text { Credit }\end{array}$ & $\begin{array}{c}\text { Bonds and } \\
\text { Exchange } \\
\text { Rates }\end{array}$ & Prices & $\begin{array}{c}\text { Stock } \\
\text { Market }\end{array}$ \\
\hline \multicolumn{9}{|c|}{ Lasso } \\
\hline Output & 0.025 & 0.040 & 0.012 & 0.059 & 0.016 & 0.021 & 0.001 & 0.004 \\
\hline Labor & 0.024 & 0.094 & 0.025 & 0.048 & 0.010 & 0.018 & 0.007 & 0.030 \\
\hline Housing & 0.009 & 0.024 & 0.218 & 0.008 & 0.052 & 0.051 & 0.002 & 0.064 \\
\hline Consumption & 0.022 & 0.030 & 0.045 & 0.107 & 0.017 & 0.018 & 0.006 & 0.019 \\
\hline Money & 0.012 & 0.018 & 0.009 & 0.020 & 0.137 & 0.037 & 0.016 & 0.026 \\
\hline Bonds & 0.007 & 0.028 & 0.021 & 0.035 & 0.007 & 0.079 & 0.023 & 0.081 \\
\hline Prices & 0.016 & 0.003 & 0.002 & 0.033 & 0.033 & 0.012 & 0.077 & 0.000 \\
\hline Stock & 0.000 & 0.007 & 0.000 & 0.039 & 0.024 & 0.073 & 0.010 & 0.138 \\
\hline \multicolumn{9}{|c|}{ Adaptive Lasso } \\
\hline Output & 0.011 & 0.013 & 0.000 & 0.104 & 0.065 & 0.002 & 0.020 & 0.001 \\
\hline Labor & 0.012 & 0.025 & 0.002 & 0.100 & 0.070 & 0.002 & 0.020 & 0.001 \\
\hline Housing & 0.036 & 0.051 & 0.059 & 0.233 & 0.095 & 0.014 & 0.039 & 0.006 \\
\hline Consumption & 0.009 & 0.012 & 0.001 & 0.078 & 0.048 & 0.002 & 0.017 & 0.001 \\
\hline Money & 0.001 & 0.002 & 0.001 & 0.009 & 0.022 & 0.001 & 0.003 & 0.000 \\
\hline Bonds & 0.018 & 0.019 & 0.001 & 0.108 & 0.059 & 0.007 & 0.023 & 0.004 \\
\hline Prices & 0.001 & 0.001 & 0.000 & 0.012 & 0.010 & 0.000 & 0.005 & 0.000 \\
\hline Stock & 0.000 & 0.003 & 0.000 & 0.024 & 0.083 & 0.000 & 0.021 & 0.000 \\
\hline \multicolumn{9}{|c|}{ Adaptive Group Lasso } \\
\hline Output & 0.043 & 0.000 & 0.002 & 0.175 & 0.014 & 0.000 & 0.000 & 0.005 \\
\hline Labor & 0.001 & 0.056 & 0.070 & 0.103 & 0.000 & 0.000 & 0.000 & 0.023 \\
\hline Housing & 0.000 & 0.000 & 0.009 & 0.000 & 0.000 & 0.000 & 0.000 & 0.000 \\
\hline Consumption & 0.000 & 0.000 & 0.000 & 0.210 & 0.000 & 0.000 & 0.000 & 0.014 \\
\hline Money & 0.000 & 0.000 & 0.006 & 0.000 & 0.267 & 0.000 & 0.000 & 0.006 \\
\hline Bonds & 0.000 & 0.000 & 0.038 & 0.011 & 0.000 & 0.115 & 0.000 & 0.090 \\
\hline Prices & 0.000 & 0.000 & 0.000 & 0.000 & 0.020 & 0.000 & 0.273 & 0.000 \\
\hline Stock & 0.000 & 0.000 & 0.000 & 0.000 & 0.000 & 0.000 & 0.000 & 0.206 \\
\hline
\end{tabular}

TABLE 5.3. Selection frequency, average over 96 forecasts at horizon 1. Largest share of selected variables in bold. 


\begin{tabular}{|c|c|c|c|c|c|c|c|c|c|}
\hline & $\begin{array}{l}\text { Output } \\
\text { and } \\
\text { Income }\end{array}$ & $\begin{array}{c}\text { Labor } \\
\text { Market }\end{array}$ & Housing & $\begin{array}{c}\text { Consumption } \\
\text { Orders } \\
\text { Inventories }\end{array}$ & $\begin{array}{l}\text { Money } \\
\text { and } \\
\text { Credit }\end{array}$ & $\begin{array}{c}\text { Bonds and } \\
\text { Exchange } \\
\text { Rates }\end{array}$ & Prices & $\begin{array}{c}\text { Stock } \\
\text { Market }\end{array}$ & Total \\
\hline \multicolumn{10}{|c|}{ Lasso } \\
\hline Output & 0.833 & 2.591 & 0.238 & 1.664 & 0.354 & 0.919 & 0.037 & 0.030 & 6.665 \\
\hline Labor & 0.804 & 6.034 & 0.506 & 1.340 & 0.223 & 0.779 & 0.275 & 0.236 & 10.196 \\
\hline Housing & 0.301 & 1.557 & 4.356 & 0.227 & 1.151 & 2.227 & 0.086 & 0.512 & 10.419 \\
\hline Consumption & 0.754 & 1.911 & 0.900 & 3.006 & 0.369 & 0.790 & 0.256 & 0.155 & 8.141 \\
\hline Money & 0.403 & 1.149 & 0.176 & 0.565 & 3.023 & 1.606 & 0.655 & 0.209 & 7.787 \\
\hline Bonds & 0.244 & 1.773 & 0.419 & 0.974 & 0.145 & 3.473 & 0.973 & 0.647 & 8.647 \\
\hline Prices & 0.542 & 0.211 & 0.036 & 0.936 & 0.728 & 0.537 & 3.224 & 0.001 & 6.216 \\
\hline Stock & 0.003 & 0.427 & 0.000 & 1.081 & 0.526 & 3.208 & 0.419 & 1.107 & 6.771 \\
\hline \multicolumn{10}{|c|}{ Adaptive Lasso } \\
\hline Output & 0.385 & 0.857 & 0.009 & 2.920 & 1.420 & 0.072 & 0.857 & 0.011 & 6.531 \\
\hline Labor & 0.422 & 1.586 & 0.033 & 2.807 & 1.546 & 0.093 & 0.860 & 0.007 & 7.353 \\
\hline Housing & 1.207 & 3.275 & 1.172 & 6.511 & 2.092 & 0.636 & 1.641 & 0.050 & 16.584 \\
\hline Consumption & 0.295 & 0.768 & 0.018 & 2.187 & 1.066 & 0.068 & 0.708 & 0.009 & 5.119 \\
\hline Money & 0.023 & 0.100 & 0.027 & 0.243 & 0.486 & 0.038 & 0.117 & 0.004 & 1.039 \\
\hline Bonds & 0.597 & 1.230 & 0.029 & 3.031 & 1.298 & 0.330 & 0.950 & 0.031 & 7.497 \\
\hline Prices & 0.018 & 0.056 & 0.000 & 0.333 & 0.230 & 0.003 & 0.189 & 0.001 & 0.831 \\
\hline Stock & 0.010 & 0.174 & 0.000 & 0.682 & 1.826 & 0.005 & 0.865 & 0.000 & 3.562 \\
\hline \multicolumn{10}{|c|}{ Adaptive Group Lasso } \\
\hline Output & 1.478 & 0.000 & 0.037 & 4.904 & 0.310 & 0.000 & 0.000 & 0.039 & 6.768 \\
\hline Labor & 0.044 & 3.556 & 1.405 & 2.873 & 0.007 & 0.000 & 0.000 & 0.186 & 8.072 \\
\hline Housing & 0.000 & 0.000 & 0.188 & 0.000 & 0.000 & 0.000 & 0.000 & 0.000 & 0.188 \\
\hline Consumption & 0.000 & 0.000 & 0.000 & 5.874 & 0.000 & 0.000 & 0.000 & 0.112 & 5.986 \\
\hline Money & 0.000 & 0.000 & 0.114 & 0.000 & 5.884 & 0.000 & 0.000 & 0.045 & 6.043 \\
\hline Bonds & 0.000 & 0.000 & 0.758 & 0.318 & 0.000 & 5.057 & 0.000 & 0.717 & 6.850 \\
\hline Prices & 0.000 & 0.000 & 0.000 & 0.000 & 0.436 & 0.000 & 11.476 & 0.004 & 11.916 \\
\hline Stock & 0.000 & 0.000 & 0.000 & 0.000 & 0.000 & 0.000 & 0.000 & 1.646 & 1.646 \\
\hline
\end{tabular}

TABLE 5.4. Average number of variables per equation, average over 96 forecasts at horizon 1. Largest number of selected variables in bold. 


\begin{tabular}{|c|c|c|c|c|c|c|c|c|}
\hline & $\begin{array}{l}\text { Output } \\
\text { and } \\
\text { Income }\end{array}$ & $\begin{array}{l}\text { Labor } \\
\text { Market }\end{array}$ & Housing & $\begin{array}{l}\text { Consumption } \\
\text { Orders } \\
\text { Inventories }\end{array}$ & $\begin{array}{l}\text { Money } \\
\text { and } \\
\text { Credit }\end{array}$ & $\begin{array}{c}\text { Bonds and } \\
\text { Exchange } \\
\text { Rates }\end{array}$ & Prices & $\begin{array}{l}\text { Stock } \\
\text { Market }\end{array}$ \\
\hline \multicolumn{9}{|c|}{ Recursive Forecasts } \\
\hline Lasso & 0.6606 & 0.7688 & 0.6632 & 0.7184 & 0.6197 & 0.5155 & 0.7497 & 0.7970 \\
\hline aLasso & 0.7070 & 0.8781 & 6.5737 & 0.7826 & 0.5902 & 1.2767 & 0.7368 & 0.8048 \\
\hline agLasso & 0.7397 & 0.9911 & 8.1884 & 1.0178 & 0.6170 & 1.5313 & 0.7956 & 0.7871 \\
\hline \multicolumn{9}{|c|}{ Direct Forecasts } \\
\hline OLS-VAR & 2.2095 & 1.8309 & 1.2194 & 1.4316 & 1.3817 & 1.5667 & 1.2341 & 2.0118 \\
\hline Lasso & 0.6830 & 0.7115 & 0.5980 & 0.7175 & 0.6019 & 0.5593 & 0.7364 & 0.7846 \\
\hline aLasso & 0.7277 & 0.9519 & 6.5100 & 0.8264 & 0.6080 & 1.2070 & 0.7446 & 0.8041 \\
\hline agLasso & 0.7395 & 0.9901 & 8.1591 & 1.0169 & 0.6171 & 1.5317 & 0.7957 & 0.7873 \\
\hline \multicolumn{9}{|c|}{ Factor model forecasts } \\
\hline $\mathrm{CF} 1$ & 0.6945 & 0.7585 & 0.9643 & 0.8606 & 0.5852 & 0.5875 & 0.7680 & 0.8184 \\
\hline $\mathrm{CF} 2$ & 0.6868 & 0.7396 & 1.0159 & 0.8293 & 0.5872 & 0.5739 & 0.7429 & 0.8775 \\
\hline $\mathrm{CF} 3$ & 0.6818 & 0.7496 & 1.0141 & 0.7902 & 0.5892 & 0.5708 & 0.7440 & 0.8810 \\
\hline $\mathrm{CF} 4$ & 0.6737 & 0.7514 & 0.9959 & 0.7784 & 0.5896 & 0.5663 & 0.7448 & 0.8678 \\
\hline CF 5 & 0.7024 & 0.7562 & 1.0058 & 0.7823 & 0.5886 & 0.5655 & 0.7532 & 0.8709 \\
\hline $\mathrm{CF} \mathrm{BIC}$ & 0.6667 & 0.7438 & 0.9726 & 0.8422 & 0.5822 & 0.6388 & 0.7626 & 0.8363 \\
\hline \multicolumn{9}{|c|}{ Univariate forecasts } \\
\hline LSTAR & 0.7334 & 0.7178 & 0.8210 & 0.7918 & 1.1548 & 0.5436 & 16.2912 & 1.0083 \\
\hline $\operatorname{AR}(1)$ & 1.1481 & 1.0042 & 0.8365 & 1.1427 & 0.9493 & 0.8433 & 1.4706 & 1.5428 \\
\hline
\end{tabular}

TABLE 5.5. MSE relative to the recursive VAR MSE 3 step ahead forecasts, 94 forecasts

\begin{tabular}{|c|c|c|c|c|c|c|c|c|}
\hline & $\begin{array}{l}\text { Output } \\
\text { and } \\
\text { Income }\end{array}$ & $\begin{array}{l}\text { Labor } \\
\text { Market }\end{array}$ & Housing & $\begin{array}{c}\text { Consumption } \\
\text { Orders } \\
\text { Inventories }\end{array}$ & $\begin{array}{l}\text { Money } \\
\text { and } \\
\text { Credit }\end{array}$ & $\begin{array}{c}\text { Bonds and } \\
\text { Exchange } \\
\text { Rates }\end{array}$ & Prices & $\begin{array}{l}\text { Stock } \\
\text { Market }\end{array}$ \\
\hline \multicolumn{9}{|c|}{ Recursive Forecasts } \\
\hline Lasso & 0.5832 & 0.7370 & 0.6447 & 0.6178 & 0.4571 & 0.5671 & 0.5597 & 0.9042 \\
\hline aLa & 0.6231 & 0.8004 & 3.8090 & & 0.4479 & & & 0.9007 \\
\hline agLa & 0.6010 & 0.7900 & 4.4731 & 0.7246 & 0.4630 & 1.0267 & 0.5862 & 0.8985 \\
\hline \multicolumn{9}{|c|}{ Direct Forecasts } \\
\hline OLS-VAR & 1.1667 & 1.4914 & 1.1594 & 1.3400 & 0.7106 & 6 & 0.9 & 1.6656 \\
\hline Lasso & 0.6258 & 0.7393 & 0.5105 & 0.6802 & 0.4519 & 80 & 94 & 0.9033 \\
\hline aLasso & 0.6846 & 0.8297 & 4.2371 & 0.7826 & 0.4612 & 0.8562 & 0.5854 & 0.9122 \\
\hline agLasso & 0.6002 & 0.7871 & 4.4401 & 0.7225 & 0.4632 & 1.0279 & 0.5862 & 0.8985 \\
\hline \multicolumn{9}{|c|}{ Factor model forecasts } \\
\hline CF 1 & 0.6225 & 0.6 & 0.9298 & 0.7093 & 0.4204 & & 0.6052 & 0.9555 \\
\hline $\mathrm{CF} 2$ & 0.6260 & 0.69 & 1.0067 & & 0.4200 & & & 1.0185 \\
\hline $\mathrm{CF}$ & 0.6128 & 0.6 & & & & & & 1.0140 \\
\hline $\mathrm{CF}$ & 6999 & & & & & & & 90 \\
\hline $\mathrm{CF}$ & & & & & & & & \\
\hline $\mathrm{CF} \mathrm{BIC}$ & 6083 & 12 & 0.9449 & 0.6827 & 0.4522 & & 975 & .0131 \\
\hline \multicolumn{9}{|c|}{ Univariate forecasts } \\
\hline & 0.6442 & $0.67 \varepsilon$ & 0.9285 & 0.6719 & 0.5898 & & & 1.0313 \\
\hline $\operatorname{AR}(1)$ & 1.0351 & 0.9905 & 0.8884 & 0.9904 & 0.7726 & 0.9432 & 1.1250 & 1.6410 \\
\hline
\end{tabular}

TABLE 5.6. MSE relative to the recursive VAR MSE, 6 step ahead forecasts, 91 forecasts 


\begin{tabular}{|c|c|c|c|c|c|c|c|c|}
\hline & $\begin{array}{l}\text { Output } \\
\text { and } \\
\text { Income }\end{array}$ & $\begin{array}{l}\text { Labor } \\
\text { Market }\end{array}$ & Housing & $\begin{array}{c}\text { Consumption } \\
\text { Orders } \\
\text { Inventories }\end{array}$ & $\begin{array}{l}\text { Money } \\
\text { and } \\
\text { Credit }\end{array}$ & $\begin{array}{c}\text { Bonds and } \\
\text { Exchange } \\
\text { Rates }\end{array}$ & Prices & $\begin{array}{l}\text { Stock } \\
\text { Market }\end{array}$ \\
\hline \multicolumn{9}{|c|}{ Recursive Forecasts } \\
\hline Lasso & 0.2652 & 0.3111 & 0.4014 & 0.2562 & 0.2693 & 0.3544 & 0.1788 & 0.7923 \\
\hline aLas & 0.2713 & 0.3 & 1.3618 & & 0.2665 & & & \\
\hline agLasso & 0.2654 & 0.3074 & 1.4246 & & 0.2695 & 5 & 0.1879 & 0.7916 \\
\hline \multicolumn{9}{|c|}{ Direct Forecasts } \\
\hline OLS-VAR & 0.7486 & 1.0626 & 0.8729 & 0.8459 & 0.5968 & & 0.5 & 2.7719 \\
\hline Lasso & 0.2769 & 0.3193 & 0.3 & 0.3 & 0.2746 & & 00 & 0.7907 \\
\hline $\mathrm{aLa}$ & 0.3374 & 0.4007 & 34 & 0.4 & 0.2736 & & 0.2287 & .8172 \\
\hline agLasso & 0.2644 & 0.3052 & 1.3988 & 0.2660 & 0.2697 & 0.4617 & 0.1879 & 0.7917 \\
\hline \multicolumn{9}{|c|}{ Factor model forecasts } \\
\hline $\mathrm{CF} 1$ & 0.2619 & 0.3052 & 0.5581 & 0.2915 & 0.2572 & 0.3512 & 0.1938 & 0.8532 \\
\hline $\mathrm{CF} 2$ & 0.2704 & 0.3137 & 0.5844 & 0.2963 & 0.2566 & & 0.1926 & 0.9023 \\
\hline $\mathrm{CF} 3$ & 0.2735 & 0.3165 & 0.5 & 0.3 & & & 0.1924 & 0.9054 \\
\hline $\mathrm{CF} 4$ & 0.2673 & 0.3054 & & & & & & \\
\hline CF 5 & 0.2690 & 0 & & & & & & 34 \\
\hline $\mathrm{CF} \mathrm{BIC}$ & 0.2864 & 0 & 699 & 0.3245 & 0.8 & & & \\
\hline \multicolumn{9}{|c|}{ Univariate forecasts } \\
\hline & 0 & & & & & & & \\
\hline $\operatorname{AR}(1)$ & 0.5152 & 0.5325 & 0.5862 & 0.5007 & 0.5440 & 0.6828 & 0.4152 & 1.6023 \\
\hline
\end{tabular}

TABLE 5.7. MSE relative to the recursive VAR MSE, 12 step ahead forecasts, 85 forecasts 


\begin{tabular}{|c|c|c|c|c|c|c|c|c|}
\hline & $\begin{array}{l}\text { Output } \\
\text { and } \\
\text { Income }\end{array}$ & $\begin{array}{l}\text { Labor } \\
\text { Market }\end{array}$ & Housing & $\begin{array}{c}\text { Consumption } \\
\text { Orders } \\
\text { Investment }\end{array}$ & $\begin{array}{l}\text { Money } \\
\text { and } \\
\text { Credit }\end{array}$ & $\begin{array}{c}\text { Bonds and } \\
\text { Exchange } \\
\text { Rates }\end{array}$ & Prices & $\begin{array}{l}\text { Stock } \\
\text { Market }\end{array}$ \\
\hline \multicolumn{9}{|c|}{ Direct Lasso } \\
\hline Output & 0.004 & 0.003 & 0.006 & 0.023 & 0.017 & 0.027 & 0.008 & 0.000 \\
\hline Labor & 0.001 & 0.016 & 0.022 & 0.018 & 0.022 & 0.042 & 0.005 & 0.007 \\
\hline Housing & 0.008 & 0.083 & 0.216 & 0.082 & 0.092 & 0.131 & 0.002 & 0.003 \\
\hline Consumption & 0.006 & 0.012 & 0.059 & 0.011 & 0.043 & 0.030 & 0.004 & 0.005 \\
\hline Money & 0.001 & 0.007 & 0.003 & 0.015 & 0.017 & 0.006 & 0.005 & 0.016 \\
\hline Bonds & 0.012 & 0.015 & 0.070 & 0.016 & 0.017 & 0.035 & 0.018 & 0.056 \\
\hline Prices & 0.001 & 0.005 & 0.011 & 0.000 & 0.007 & 0.001 & 0.005 & 0.003 \\
\hline Stock & 0.002 & 0.000 & 0.000 & 0.003 & 0.000 & 0.000 & 0.000 & 0.000 \\
\hline \multicolumn{9}{|c|}{ Direct Adaptive Lasso } \\
\hline Output & 0.001 & 0.001 & 0.000 & 0.010 & 0.040 & 0.000 & 0.011 & 0.000 \\
\hline Labor & 0.006 & 0.007 & 0.000 & 0.022 & 0.045 & 0.000 & 0.012 & 0.000 \\
\hline Housing & 0.016 & 0.022 & 0.016 & 0.078 & 0.072 & 0.002 & 0.027 & 0.000 \\
\hline Consumption & 0.006 & 0.007 & 0.000 & 0.019 & 0.036 & 0.000 & 0.013 & 0.000 \\
\hline Money & 0.001 & 0.000 & 0.000 & 0.001 & 0.018 & 0.000 & 0.001 & 0.000 \\
\hline Bonds & 0.001 & 0.004 & 0.0 & 0.011 & & 0.0 & 0.012 & 0.000 \\
\hline Prices & 0.001 & 0 & $0 .($ & & & & 02 & 0.000 \\
\hline Stock & 0.002 & 0.001 & 0.000 & 0.001 & 0.014 & $0 .($ & 0.000 & 0.000 \\
\hline \multicolumn{9}{|c|}{ Direct Adaptive Group Lasso } \\
\hline Output & 0.034 & 0.000 & 0.004 & 0.094 & 0.037 & 0.033 & 0.000 & 0.006 \\
\hline Labor & 0.000 & 0.025 & 0.086 & 0.042 & 0.008 & 0.009 & 0.020 & 0.005 \\
\hline Housing & 0.000 & 0.000 & 0.000 & 0.000 & 0.000 & 0.000 & 0.000 & 0.000 \\
\hline Consumption & 0.001 & 0.000 & 0.059 & 0.046 & 0.034 & 0.009 & 0.017 & 0.004 \\
\hline Money & 0.000 & 0.001 & 0.026 & 0.034 & 0.034 & 0.006 & 0.032 & 0.018 \\
\hline Bonds & 0.000 & 0.001 & 0.091 & 0.021 & 0.020 & 0.109 & 0.000 & 0.004 \\
\hline Prices & 0.016 & 0.000 & 0.007 & 0.013 & 0.041 & 0.000 & 0.080 & 0.004 \\
\hline Stock & 0.000 & 0.000 & 0.000 & 0.012 & 0.029 & 0.065 & 0.000 & 0.015 \\
\hline
\end{tabular}

TABLE 5.8. Selection frequency, average over 85 forecasts at horizon 12. Most selected group in bold. 
2012-22: $\quad$ Peter O. Christensen and Zhenjiang Qin: Information and Heterogeneous Beliefs: Cost of Capital, Trading Volume, and Investor Welfare

2012-23: $\quad$ Zhenjiang Qin: Heterogeneous Beliefs, Public Information, and Option Markets

2012-24: $\quad$ Zhenjiang Qin: Continuous Trading Dynamically Effectively Complete Market with Heterogeneous Beliefs

2012-25: Heejoon Han and Dennis Kristensen: Asymptotic Theory for the QMLE in GARCH-X Models with Stationary and Non-Stationary Covariates

2012-26: Lei Pan, Olaf Posch and Michel van der Wel: Measuring Convergence using Dynamic Equilibrium Models: Evidence from Chinese Provinces

2012-27: $\quad$ Lasse Bork and Stig V. Møller: Housing price forecastability: A factor analysis

2012-28: Johannes Tang Kristensen: Factor-Based Forecasting in the Presence of Outliers: Are Factors Better Selected and Estimated by the Median than by The Mean?

2012-29: $\quad$ Anders Rahbek and Heino Bohn Nielsen: Unit Root Vector Autoregression with volatility Induced Stationarity

2012-30: $\quad$ Eric Hillebrand and Marcelo C. Medeiros: Nonlinearity, Breaks, and Long-Range Dependence in Time-Series Models

2012-31: $\quad$ Eric Hillebrand, Marcelo C. Medeiros and Junyue Xu: Asymptotic Theory for Regressions with Smoothly Changing Parameters

2012-32: $\quad$ Olaf Posch and Andreas Schrimpf: Risk of Rare Disasters, Euler Equation Errors and the Performance of the C-CAPM

2012-33: Charlotte Christiansen: Integration of European Bond Markets

2012-34: Nektarios Aslanidis and Charlotte Christiansen: Quantiles of the Realized Stock-Bond Correlation and Links to the Macroeconomy

2012-35: Daniela Osterrieder and Peter C. Schotman: The Volatility of Longterm Bond Returns: Persistent Interest Shocks and Time-varying Risk Premiums

2012-36: Giuseppe Cavaliere, Anders Rahbek and A.M.Robert Taylor: Bootstrap Determination of the Co-integration Rank in Heteroskedastic VAR Models

2012-37: $\quad$ Marcelo C. Medeiros and Eduardo F. Mendes: Estimating HighDimensional Time Series Models

2012-38: $\quad$ Anders Bredahl Kock and Laurent A.F. Callot: Oracle Efficient Estimation and Forecasting with the Adaptive LASSO and the Adaptive Group LASSO in Vector Autoregressions 